belegen, kann er zwar nicht vorweisen, aber die fehlenden Schwankungen im FEV1 könnten sehr wohl darauf zurückzuführen sein, daß es bereits zu einer Fibrose der Atemwege gekommen ist. Sie sind ein typisches histologisches Kennzeichen bei Patienten, die im Status asthmaticus gestorben sind.

\section{Typisch für,,Chaoten“: Die stark schwankende Lungenfunktion}

Neben den Non-Chaoten gibt es, der Name deutet es ja bereits an, eine zweite Gruppe von Patienten mit „chaotischen“ Ausschlägen in den Werten der Sekundenkapazität. Die FEV1 zeigte über den Tag hinweg deutlich stärkere Schwankungen als beim Gesunden. Definiert wurde dieser Typ als eine mehr als 30\%ige Variabilität der FEV1-Werte, während die Variabilität bei den Non-Chaoten definitionsgemäß bei unter $15 \%$ lag.

Typisches Kennzeichen der SI bei den „Chaoten“ war, daß die Schwankungen der FEV1 unter der Steroidtherapie keine Änderungen zeigten. Die Ursache ist unklar. Die Prognose ist jedoch vermutlich besser als bei der non-chaotischen Form, da die chaotischen Patienten teilweise auch normale FEV1-Werte erreichen. Es ist deshalb unwahrscheinlich, daß es bereits zu irreversiblen Veränderungen der Atemwege gekommen ist.

Als Maßnahmen zur Vermeidung eines Status asthmaticus schlagen die Autoren vor, sehr sorgfältig nach Triggern zu suchen, welche die Lungenfunktion verschlechtern könnten. An der Klinik werden die Patienten außerdem durch ausgebildetes Fach- personal in der richtigen Inhalationstechnik geschult. Außerdem müsse die Compliance der Patienten sichergestellt werden. In Denver müssen die Patienten die Medikamente deshalb unter Aufsicht einnehmen. (rme)

Quelle: Chan MT et al.: Difficult-to-control asthma: clinical characteristics of steroid-insensitive asthma. J Allergy Clin

\title{
Welches Therapieregime bei Krupp?
}

\section{Zur Behandlung des Krupps ist die orale Gabe von Dexamethason aufgrund der einfachen Handhabbarkeit und der geringen Kosten Therapie der ersten Wahl.}

D ie Wirksamkeit von Kortikoidpräparaten zur Behandlung des Krupps steht heute außer Zweifel. Unklar ist jedoch noch immer, welchem Glukokortikoid und welcher Applikationsform der Vorzug gegeben werden sollte. Eine Antwort auf diese Frage suchte eine kanadische Arbeitsgruppe im Rahmen einer randomisierten, kontrollierten klinischen Studie.

\section{Arme Asthmakinder}

Eine Untersuchung an Münchner Schulen zeigt: Kinder aus den unteren sozialen Schichten leiden signifikant häufiger unter schwerem Asthma bronchiale als Kinder aus gesicherten Verhältnissen.

Die Wissenschaftler des Forschungszentrums für Umwelt und Gesundheit (GSF) in Neuherberg bei München untersuchten zwei Jahre lang 4434 Münchner Schüler im Alter zwischen 9 und 11 Jahren. Dabei zeigte sich, daß schweres Asthma in der unteren sozialen Schicht etwa 2,4mal häufiger vorkommt als bei Kindern der oberen sozialen Schicht.

Für die Zugehörigkeit zu den sozialen Schichten war die Schulbildung der Eltern maßgeblich: Hauptschulabsolventen wurden der unteren, Eltern mit Realschulabschluß der mittleren und Eltern mit Abitur der oberen sozialen Schicht zugeordnet. Die Einteilung des Asthmaschweregrades erfolgte in drei Gruppen: 1 bis 4 Anfälle in den vergangenen zwölf Monaten wurden als leichtes Asthma eingestuft, 5 bis 10 Attacken galten als mittleres und mehr als 10 als schweres Asthma.

Das Resümee der Wissenschaftler: Der Früherkennung und Therapie des Asthmas sollte vor allem bei Kindern aus einfachen Familienverhältnissen noch mehr Aufmerksamkeit geschenkt werden.

Kinder im Alter zwischen drei Monaten und fünf Jahren mit einem KruppScore über 2 wurden in die Studie aufgenommen. Nach randomisierter $\mathrm{Zu}$ teilung erhielten 64 Kinder $0,6 \mathrm{mg} / \mathrm{kg}$ Dexamethason oral zusammen mit einer Budesonid-Inhalation (2 mg), 69 wurden nur mit Dexamethason behandelt und inhalierten Plazebo, 66 weitere erhielten oral ein Plazebopräparat und Budesonid zur Inhalation.

Die klinischen Ergebnisse unter diesen drei verschiedenen Regimes unterschieden sich kaum: Im Westley Krupp-Score wurde durch Budesonid allein eine Reduktion um 2,3 Punkte erzielt, unter Dexamethason um 2,4 und unter der Kombination ebenfalls um 2,4 Punkte.

\section{Im Zweifelsfall Dexamethason}

Auch bezüglich weiterer Parameter wie stationäre Aufnahme, Notwendigkeit von Arztbesuchen u.ä. fanden sich keine statistisch signifikanten Unterschiede zwischen den drei Gruppen.

Angesichts dieser Ergebnisse empfehlen die Autoren zur Initialtherapie des Krupps entweder die alleinige Gabe von Dexamethason p.o. oder die Inhalation von Budesonid. Kostenerwägungen und die leichte Handhabbarkeit könnten im Zweifelsfall den Ausschlag für Dexamethason geben. (mo)

Quelle: Klassen TP et al.: Nebulized Budesonide and Oral Dexamethasone for Treatment of Croup. A Randomized Controlled Trial. J Amer med Ass 1998; 279: 1629-32. 